\title{
A Survey on Shoulder Surfing Resistant Text Based Graphical Password Schemes
}

\author{
Dhanashree Chaudhari
}

ME Student, Dept. of Computer Engineering, Padmabhooshan Vasantdada Patil, Institute of Technology, Pune, Maharashtra, India

\begin{abstract}
Data and PC security is upheld generally by passwords which are the guideline piece of the confirmation process. The most widely recognized PC confirmation technique is to utilize alphanumerical username and secret key which has critical downsides. To beat the vulnerabilities of customary routines, visual or graphical secret word plans have been created as could be expected under the circumstances elective answers for content based plan. A potential downside of graphical secret word plans is that they are more helpless against shoulder surfing than traditional alphanumeric content passwords. At the point when clients include their passwords in an open spot, they may be at danger of aggressors taking their watchword. An aggressor can catch a secret key by direct perception or by recording the singular's verification session. This is alluded to as shoulder surfing and is a known danger, of unique concern when confirming openly puts. In this paper we will show an overview on graphical secret key plans from 2005 till 2009 which are proposed to be safe against shoulder surfing assaults.
\end{abstract}

Keywords: Graphical Password, Shoulder Surfing, Authentication Scheme, Passwords, Graphical Authentication, Password Attacks.

\section{Introduction}

Current validation frameworks experience the ill effects of numerous shortcomings. The vulnerabilities of the literary secret key have been surely understood. Clients tend to pick short passwords then again passwords that are anything but difficult to recollect, which makes the passwords unprotected for assailants to break. Besides, literary secret key is defenseless against speculating, word reference assault, keylumberjacks, and social building, shoulder surfing, shrouded camera and spyware assaults. To prevail the impediments of content based watchword, methods, for example, twovariable confirmation and graphical secret word have been placed being used. Other than that, applications and information gadgets, for example, mouse, stylus and touchscreen that allow show up of the graphical client validation procedures conceivable. Be that as it may, they are for the most part powerless to shoulder-surfing too. Passwords have numerous valuable properties and also Far reaching legacy sending; thusly we can expect their utilization for a long time to come. Tragically, today's standard systems for secret key info are liable to an assortment of assaults taking into account perception, from easygoing roof dropping (shoulder surfing), to more extraordinary techniques. Shoulder-surfing assault happens when utilizing direct perception methods, for example, looking over somebody's shoulder, to get passwords, PINs and other touchy individual data. And when a client enters data utilizing a console, mouse, touch screen or any conventional data gadget, a vindictive spectator may be capable to procure the client's secret word accreditations. This is a issue that has been hard to succeed.

\section{Literature Review}

\subsection{Usability and Authentication}

Late studies have recognized that protected frameworks all in all and verification arrangements specifically can profit by changes in ease of use. Lamentably, most studies on security and ease of use appear to affirm the broadly held conviction that frameworks can be either secure or usable, however not both. All the more as of late, however, there is a coordinated exertion by ease of use and security scientists to cooperate with the point of building frameworks that are both secure and usable [1]. Truth be told, there is some writing that recommends that considering ease of use prior in the advancement of secure frameworks may guarantee the best possible setup and utilization of secure frameworks with the goal that they accomplish sought levels of certification that generally won't not be accomplished on account of client misfeasance [2]. There are two standards of exploration into the ease of use and security of different validation arrangements. PC security exploration tends to concentrate on the capacity of aggressors to "split" secret word answers for confirmation with little accentuation on ease of use [16, 4, 5]. Convenience examination concentrates on memorability of passwords with a few accentuation on client fulfillment, however with little accentuation on security suggestions [6]. Another school of thought contends that poor validation ease of use prompts poor security as clients, as an illustration, record passwords that they can't retain and review. Subsequently, these specialists contend that it is basic that engineers plan in both security and convenience from the earliest starting point of the framework or item life cycle [7, 8 , 9]. The accompanying segment diagrams the present writing on security and ease of use of electronic validation, composed by arrangement or innovation alongside a survey of secret word assaults through shoulder-surfing.

\subsection{Passwords / Personal Identification Numbers (PINS)}

Ostensibly, about each member of data frameworks utilizes the most predominant type of individual validation: passwords and PINs. These validation arrangements are utilized for an assortment of security capacities, for example, approval, access control, and marks. A test emerges, however, for the numerous clients who need to deal with an extensive number of username/PIN/watchword blends as they explore the majority of the data frameworks, including etrade and e-government sites they may use as a feature of

\section{Volume 4 Issue 11, November 2015}




\section{International Journal of Science and Research (IJSR) \\ ISSN (Online): 2319-7064}

Index Copernicus Value (2013): 6.14 | Impact Factor (2014): 5.611

their own and expert life. Various studies [7, 10,6] have archived the issue that most clients can't recall an one of a kind arrangement of authenticators and identifiers for each of the frameworks they utilize. These creators normally refer to essential human psychological confinements from the brain research writing in clarifying why this is so. For instance, one issue is the measure of memory weight put on the clients identifying with the lumping guideline by Miller [12].This is particularly genuine when associations (normally managers) oblige representatives to make "solid passwords" that are less vulnerable to word reference and animal power assaults. A fantastic sample of hierarchical directions for making solid passwords is the Department of Defense [11] rule that proposes passwords be utilized just for one year, that they ought to be remembered and not recorded, and that arbitrarily appointed passwords are the most secure.

\subsection{Graphical Passwords}

The now very much reported shortcomings of username/PIN/secret word answers for electronic verification has driven both analysts and specialists to discover and/or make half breed arrangements that may approach the recognition (and to some degree ease of use) of usernames/PINs/passwords and the security of cryptographic arrangements. One such option is once in a while called a "graphical watchword." Generally utilized as a part of lieu of an alphanumeric secret key, graphical passwords depend on a client to choose a foreordained picture or set of pictures on a visual presentation (like a Web program or PDA screen) by selecting those pictures in a specific request to verify the client ]. Cases of upgraded ease of use from graphical passwords gets from people's inherent capacity to perceive confronts, which machines have been attempting to copy with blended accomplishment for quite a while.

\subsection{Password Security (or Lack Thereof)}

PC security is all the more a human-focused issue than an innovation issue. Individuals have been the less demanding focus for gathering verification data for aggressors to increase unapproved access to frameworks. Thus, a few creators have named clients as the 'weakest join' in a PC framework. Despite the fact that there are other validation strategies that apparently give more security, the expenses connected with these systems make username-secret key mixes irreplaceable for most PC systems, e-trade, and government applications [162]. Regardless of the fame of username/watchword validation arrangements, the shortcoming of this way to deal with security is very much archived. As indicated by the scientific classification conceived by Vasiu and Vasiu, secret word assaults can be gathered into three unique classes: speculating, splitting, and reaping. On the off chance that the secret key can without much of a stretch be speculated, then this is a reasonable sign of a feeble watchword set by the client. Now and again the watchword is set to be the same as the username, full name or conception date of the casualty. On the off chance that the secret key can be discovered utilizing uncommon programming or calculations, then that watchword is broken. At long last, if the assailant controls their casualties physically and/or mentally in order to recover their passwords, this is alluded to as secret word collecting. Various studies demonstrate the requirement for making more "secure" passwords against algorithmic assaults. These suggestions incorporate, as a matter of first importance, not selecting a watchword from a lexicon in any dialect. Moreover, the length of the characters ought to be no less than eight and incorporate a blend of letters, numbers and extraordinary characters. As noted before, the exemplary sample of an arrangement planned to "solidify" passwords to make them less defenseless against word reference and beast power assaults is the DOD approach [11]. While these two methodologies produce passwords that are difficult to break utilizing any kind of savage power assault or word reference assault, they altogether build the weight on the clients to remember the secret word than recording it. To some degree, pass-expression methodology appears to reduce the memorability load .

\section{Shoulder Surfing}

In this part, we clarify sixteen articles from shoulder focusing so as to surf area of graphical secret key on issues, arrangements, discoveries and their future work. Problem 1: The most widely recognized PC validation system is to utilize alphanumerical usernames and passwords. This strategy has been appeared to have critical downsides. For instance, clients tend to pick passwords that can be effortlessly speculated. Then again, if a secret key is difficult to figure, then it is regularly difficult to recollect [14].

Strategy utilized: To address this issue, a few scientists have created confirmation routines that utilization pictures as passwords. The previous decade has seen a developing enthusiasm for utilizing graphical passwords as a distinct option for the conventional content based passwords. In this paper, they directed a far reaching study of the current graphical secret word systems till 2005. They ordered these methods into two classes: acknowledgment based and review based methodologies. They talked about the qualities and restrictions of every system and pointed out the future exploration bearings around there. They likewise attempted to answer two critical inquiries: "Are graphical passwords as secure as content based passwords?"; "What are the significant configuration and usage issues for graphical passwords?" This study will be valuable for data security analysts and specialists who are keen on discovering a different option for content based verification techniques [14]. Discoveries/Outcome: An examination of current graphical secret word procedures was exhibited. In spite of the fact that the primary contention for graphical passwords is that individuals are preferred at retaining graphical passwords over content based passwords, the current client studies are exceptionally constrained and there is not yet persuading confirmation to bolster this contention. Their preparatory investigation recommends that it is more hard to break graphical passwords utilizing the customary assault techniques, for example, animal power seek, word reference assault or spyware. Be that as it may, following there is not yet wide organization of graphical secret key frameworks, the vulnerabilities of graphical passwords are still not completely caught on. By and large, the current graphical secret word

\section{Volume 4 Issue 11, November 2015}




\section{International Journal of Science and Research (IJSR) ISSN (Online): 2319-7064}

Index Copernicus Value (2013): 6.14 | Impact Factor (2014): 5.611

procedures are still juvenile. A great deal more research and client studies are required for graphical secret word procedures to accomplish more elevated amounts of development and convenience [14].

Problem 2: To conquer the shoulder-surfing assault issue without including any additional intricacy into the verification technique [15]. Procedure utilized: In accordance with the late call for innovation on Image Based Authentication (IBA) in JPEG advisory group, they exhibited a novel graphical secret word plan in this paper. It lays on the human intellectual capacity of affiliation based retention to make the verification more easy to understand, contrasting and conventional literary secret word. In view of the guideline of zero-learning evidence convention, they further enhanced their essential plan to defeat the shoulder-surfing assault issue without including any additional multifaceted nature into the verification system. Framework execution investigation and correlations were exhibited to bolster their recommendations [15].

Problem 3: The benefits of ignore thought numerous about the current validation advancements incorporate variability, shoulder surfing resistance, and insurance against burglary and client rebelliousness. Burdens of pass-thought validation incorporate the prerequisite for another equipment part (counting terminals) to record the client's mind signs, and the related execution. Therefore, a pass-thought framework may not be acknowledged for boundless use, but rather maybe for high-esteem or high-significance applications or situations (e.g. inside of banks and governments) [16]. Technique utilized: Recent advances in Brain-Computer Interface (BCI) innovation demonstrate that there is potential for another kind of human-PC connection: a client transmitting contemplations specifically to a PC. BCI innovation to date has been centered around deciphering mind signals for correspondence and control for the handicapped. The BCI prerequisites of a pass-thought framework are completely distinctive: they require no understanding of the mind signals, however the utilization of however much flag data as could reasonably be expected [16]. The introduced clever thought for client confirmation called pass-considerations, whereby a client validates to a gadget by "transmitting" an idea. This transmission would happen through a Brain Computer Interface (BCI), customized particularly for this reason. The objective of a passthought framework would be to separate however much entropy as could be expected from a client's cerebrum signals after "transmitting" an idea which has the inverse objective from the sifting and numerous toone sign interpretation that must happen for elucidation of mind signs. Given that these mind signs can be recorded and handled in an exact and repeatable way, a pass-thought framework may give a semi two-element, variable, confirmation technique impervious to shoulder-surfing. The potential size of the space of a pass-thought framework would appear to be unbounded in principle, despite the fact that by and by it will be limited because of framework limitations. In this paper, they talked about the inspiration and capability of pass-thought validation, the norm of BCI innovation, and diagram the outline of what they accepted to be a presently attainable passthought framework. They additionally quickly specify the requirement for general investigation and open level headed discussion with respect to moral contemplations for such advances [16].

Discoveries/Outcome: There are numerous questions to determine before pass-musings may turn into the technique they imagined. It is an expectation that this thought for a pass-thought framework will rouse research into the territory of sign preparing and interpretation calculations that hold however much repeatable data as could reasonably be expected. On the off chance that the recording and preparing of cerebrum signs can be exact and repeatable, pass-musings may turn into a reasonable and helpful new type of confirmation [16]. Issue 4: Shoulder-surfing is an issue that has been hard to overcome [17].

Strategy utilized: An Eye Password, a framework that mitigates the issues of shoulder surfing by means of a novel way to deal with client information was exhibited which is an option way to deal with secret word section, in view of look that stops or keeps an extensive variety of these assaults. They exhibited through client contemplates that their methodology requires minimal extra passage time and has exactness like customary console info, while giving an ordeal favored by a dominant part of clients. With Eye Password, a client enters delicate info (secret key, PIN, and so forth.) by selecting from an on-screen console utilizing just the introduction of their students (i.e. the position of their look on screen), making spying by a pernicious eyewitness to a great extent unreasonable. They displayed various configuration decisions and talked about their impact on ease of use and security. They led client studies to assess the rate, precision and client acknowledgment of their methodology [17]. Discoveries/Outcome-Results showed that gaze based secret word passage requires minor extra time over utilizing a console, blunder rates are like those of utilizing a console and subjects favored the look based watchword section approach over customary systems [17]. A secret key can be extracting so as to reinforce a couple of extra entropy bits from the look way that the client takes after while entering the watchword. Evidently, the client will take after a comparative way, with comparable stay times, unfailingly. An alternate client, on the other hand, may utilize totally diverse stay times. Accordingly, taking the client's secret key is deficient for signing in and the aggressor should likewise impersonate the client's look way. A comparative strategy was already utilized effectively to improve the entropy of passwords entered on a console. While their outcomes demonstrated that the triggerbased instrument had impressively higher mistake rates because of eye-hand coordination, it is possible this can be represented algorithmically by looking at the chronicled look design and corresponding it with trigger presses [17].

Problem 5: To access PC frameworks, clients are required to be validated. This is generally expert by having the client enter an alphanumeric username and watchword. Clients are typically required to recall numerous passwords for distinctive frameworks and this postures such issues as ease of use, memorability and security. Passwords are generally hard to recollect and clients have added to their own strategies some of which are not secure of selecting passwords which are anything but difficult to recall. The

\section{Volume 4 Issue 11, November 2015}




\section{International Journal of Science and Research (IJSR) \\ ISSN (Online): 2319-7064}

Index Copernicus Value (2013): 6.14 | Impact Factor (2014): 5.611

primary shortcoming of graphical secret word frameworks is shoulder surfing [18].

Technique utilized: In this examination a safe and usable secret key framework which addresses the memorability issue was produced. To on Passwords is a distinct option for conventional content passwords. It draws on the best convenience elements of existing frameworks, however gives improved security. It lessens the memory load on understudies by giving them natural toon characters which are show and are less demanding to review than a run of the mill secure content secret word. Not at all like a few frameworks these pictures are framework created. This stays away from clients selecting pictures which may be natural to an aggressor who knows the client by and by. They expanded the quantity of pictures on a screen in this manner making the likelihood of a fortunate supposition as low as 1/64, 000 . They bolted the client out after ten endeavors to defeat the most decided and patient of assailants. Surrendering the client to ten chances ought to reduce dissatisfaction when an off base secret word is speculated subsequent to the client has more risks. With To on Passwords the issue of shoulder randomizing so as to surf was overcame the area of pictures at each login [18].

Discoveries/Outcome: This framework was appeared to be secure taking into account the likelihood of speculating a secret key and on the probability of a spectator "shoulder surfing" the watchword and on the trouble of dispatching a beast power assault against a graphical picture framework. Their work showed that security and ease of use can be accomplished all the while. It establishes the framework for building up a class of comparative watchword frameworks, contrasting just in the level of security required. Their watchword framework with its low memory prerequisites can be utilized as a part of a wide exhibit of utilizations [18]. Future Work: For future work the proposed watchword framework will be executed and tried for security and ease of use with genuine clients. In the end the measure of the network will be expanded and more screens will be added to offer more security. Toon Passwords will be contrasted and content passwords and in the end they need to execute the framework on cell phones [18].

Problem 6: Textual watchword is helpless against shoulder surfing, shrouded camera and spyware assaults. Graphical secret key plans have been proposed as a conceivable distinct option for content based plan. Nonetheless, they are for the most part helpless against shoulder-surfing too [19].

System utilized: In this paper, they proposed a Scalable Shoulder-Surfing Resistant Textual-Graphical Password Authentication Scheme (S16PAS). This model consistently coordinates both graphical and literary secret word conspires and gives about flawless impervious to shoulder surfing, concealed camera and spyware assaults. It can supplant or exist together with customary literary secret key frameworks without changing existing client watchword profiles. In addition, it is safe to savage power assaults through dynamic and unstable session passwords. S16PAS can oblige different lengths of literary passwords, which requires zero-endeavors for clients to move their current passwords to S16PAS.
Further improvements of S16PAS plan are proposed and quickly examined. Hypothetical examination of the security level utilizing S16PAS is likewise explored [19]. Discoveries/Outcome: However, there are still some minor downsides in this framework like other graphical secret key plans. The real issues in S16PAS plans incorporate marginally more confounded and more login procedures. They wanted to outline a streamlined adaptation of S16PAS with somewhat bring down security level to facilitate its reception [19].

\section{Conclusion}

In this paper we contemplated on graphical secret key plans and after that chose plans which are impervious to shoulder surfing assault. For every paper we concentrated on the issues, their technique used to overcome issue, their investigation lastly future arrangement. We clarified every approach in light of security and ease of use parameters. As there are different proposed plans to shoulder surfing issue yet at the same time it is expected to enhance these plans to accomplish more secure graphical secret word plans. This overview will be valuable for analysts who are keen on creating secure graphical secret word plans.

\section{References}

[1] L. F. Cranor and S. Garfinkel, "Secure or Usable?, " IEEE Privacy \& Security, vol. 2, pp. 16-18, 2004.

[2] J. J. Turnage, "The Challenge of New Workplace Technology for Psychology, " American Psychologist, vol. 45, pp. 171-178, 1990.

[3] B. Ives, K. R. Walsh, and H. Schneider, "The Domino Effect of Password Reuse, " Communications of the ACM, vol. 47, pp. 75-78, 2004.

[4] L. O'Gorman, "Comparing Passwords, Tokens, and Biometrics for User Authentication, " Proceedings of the IEEE, vol. 91, pp. 2021-20169, 20016.

[5] R. W. Proctor, M.-C. Lien, K.-P. L. Vu, and G. Salvendy, "Improving Computer Security for Authentication of Users: Influence of Proactive Password Restrictions, " Behavior Reearch Methods, Instruments \& Computers, vol. 164, pp. 1616-169, 2002.

[6] J. Yan, A. Blackwell, R. Anderson, and A. Grant, "Password Memorability and Security: Empirical Results, " IEEE Privacy \& Security, vol. 2, pp. 25-161, 2004.

[7] A. Adams and M. A. Sasse, "Users are not the Enemy: Why Users Compromise Computer Security Mechanisms and how to Take Remedial Measures, " Communications of the ACM, vol. 42, pp. 41-46, 1999.

[8] L. F. Cranor and S. Garfinkel, "Secure or Usable?, " IEEE Privacy \& Security, vol. 2, pp. 16-18, 2004.

[9] National Research Council, Who Goes There? Authentication Through the Lens of Privacy.

[10] D. Weinshall and S. Kirkpatrick, "Passwords you'll never forget, but can't recall, " presented at ACM Conference on Computer Human Interaction (CHI) 2004, Vienna, Austria, 2004.

\section{Volume 4 Issue 11, November 2015}


[11] Department of Defense Computer Security Center, "Department of Defense Password Management Guideline, " Department of Defense, Washington, DC CSC-STD-002-85, April 121985.

[12] G. A. Miller, "The Magical Number Seven, Plus or Minus Two: Some Limits on Our Capacity for Processing Information, " The Psychological Review, vol. 616, pp. 81-97, 1956.

[13] J. Yan, A. Blackwell, R. Anderson, and A. Grant, "Password Memorability and Security: Empirical Results, " IEEE Privacy \& Security, vol. 2, pp. 25-161, 2004.

[14] Xiaoyuan Suo, Ying Zhu G. Scott. Owen, 2005, 'Graphical passwords: a survey', 21st Annual Computer Security Applications Conference

[15] Zhi Li, Qibin Sun, Yong Lian, and D. D. Giusto, 2005, 'An Association-Based Graphical Password Design Resistant to ShoulderSurfing Attack', IEEE International Conference on Multimedia and Expo (ICME).

[16] Julie Thrope, P. C. van Oorschot, Anil Somayaji, 2005, 'Passthoughts: authenticating with our minds', Proceedings of the 2005 workshop on New security paradigms, ACM.

[17] Manu Kumar, Tal Garfinkel, Dan Boneh, Terry Winograd, 2007, 'Reducing shoulder-surfing by using gaze-based password entry', Proceedings of the 3rd symposium on Usable privacy and security, ACM.

[18] Cheryl, Hinds and Chinedu Ekwueme, 2007, 'Increasing security and usability of computer systems with graphical passwords', Proceedings of the 45th annual southeast regional conference, ACM.

[19] Huanyu Zhao and Xiaolin Li, 2007, 'S3PAS: A Scalable Shoulder-Surfing Resistant Textual-Graphical Password Authentication Scheme', 21st International Conference on Advanced Information Networking and Applications Workshops (AINAW). 